\title{
Computational Solutions for the Korteweg-deVries Equation in Warm Plasma
}

\author{
E.K. El-Shewy*, H.G. Abdelwahed, H.M. Abd-El-Hamid \\ Theoretical Physics Group \\ Faculty of Science, Mansoura University, Mansoura, Egypt \\ *e-mail:e_k_el_shewy@mans.edu.eg; emadshewy@yahoo.com
}

(Received: 5 October 2009; revised: 2 May 2010; accepted: 6 May 2010; published online: 29 June 2010)

\begin{abstract}
The reductive perturbation method has been employed to derive the Korteweg-de Vries (KdV) equation for small but finite amplitude electrostatic ion-acoustic waves. An algebraic method with computerized symbolic computation, which greatly exceeds the applicability of the existing tanh, extended tanh methods in obtaining a series of exact solutions of the KdV equation. Numerical studies have been made using plasma parameters to reveal different solutions, i.e. bell-shaped solitary pulses, rational pulses and solutions with singularity at a finite points called "blowup" solutions in addition to the propagation of explosive pulses. The result of the present investigation may be applicable to some plasma environments, such as ionosphere.
\end{abstract}

Key words: ion-acoustic waves, reductive perturbation, symbolic computations, explosive solutions

\section{INTRODUCTION}

Nonlinear phenomena play a crucial role in applied mathematics and physics. An ion-acoustic solitary wave is one of the fundamental nonlinear wave phenomena appearing in fluid dynamics [1] and plasma physics [2, 3]. There are different methods to study linear [4] and nonlinear systems [5]. Washimi and Taniti [5] were the first to use the reductive perturbation method to study the propagation of a slow modulation of quasimonochromatic waves through plasma. Numerous other authors focused on the subject [6-8]. The evolution of small but finite-amplitude solitary waves, studied by means of the KortewegdeVries $(\mathrm{KdV})$ equation, is of considerable interest in plasma dynamics.

Many powerful methods have been established and developed to study nonlinear evolution (NLEE) equations. These methods include the inverse scatting method [9], the tanh function method [10], the extended tanh method [11-13], the Exp-function method [14], the extended F-expansion method [15] and the Jacobi elliptic function expansion method [16].
There is no unified method that can be used to deal with all types of nonlinear evolution equations. Fan [17] developed a new algebraic method with computerized symbolic computation, which greatly exceeds the applicability of the existing tanh, extended tanh methods and Jacobi function expansion method in obtaining a series of exact solutions of nonlinear differential equations.

The major topic of this work is to study the solitary and other waves for ion-acoustic waves in warm plasma. This paper is organized as follows: in Section II we present the basic set of fluid equations governing our plasma model. In Section III an algorithm describing the computerized symbolic computation method is presented. In Section IV explicit solutions for $\mathrm{KdV}$ equation are obtained. Finally, some discussions and conclusions are given in section IV.

\section{BASIC EQUATIONS AND KdV EQUATION}

Consider a collisionless ionization-free unmagnetized plasma consisting of a mixture of warm ion-fluid and iso- 
thermal electrons. The basic equations describing the system in dimensionless variables is governed by [3]:

$$
\begin{gathered}
\frac{\partial n}{\partial t}+\frac{\partial(n u)}{\partial x}=0 \\
\left(\frac{\partial u}{\partial t}+u \frac{\partial u}{\partial x}\right)+\frac{\sigma}{n} \frac{\partial p}{\partial x}+\frac{\partial \phi}{\partial x}=0 \\
\left(\frac{\partial}{\partial t}+u \frac{\partial}{\partial x}\right) p+3 p \frac{\partial u}{\partial x}=0 \\
\frac{\partial^{2} \phi}{\partial x^{2}}+n-n_{e}=0 \\
n_{e}-\exp (\phi)
\end{gathered}
$$

In the earlier equations $n$ and $n_{e}$ are the densities of ions and electrons respectively, $u$ is the ion flow velocity, $p$ is the ion pressure, $\phi$ is the electric potential, $x$ is the space co-ordinate and $t$ is the time variable. $\sigma=T_{i} / T_{\mathrm{e}}<<1$ is the ratio of the ion temperature $T_{i}$ to the electron temperature $T_{e}$. All these quantities are dimensionless, being normalized in terms of the following characteristic quantities: $n$ and $n_{e}$ by the unperturbed electron density $n_{0}, u$ and $c$ by the sound velocity $\left(K T_{e} / m_{i}\right)^{1 / 2} ; p$ and $\phi$ by $n_{0} K T_{i}$ and $K T_{e} / e$ respectively; $t$ and $x$ by the inverse of the plasma frequency $\omega_{p i}^{-1}=\left(4 \pi e^{2} n_{0} / m_{i}\right)^{-1 / 2}$ and the electron Debye length $\lambda_{D}=\left(K T_{e} / 4 \pi e^{2} n_{0}\right)^{1 / 2}$ respectively. $K$ is Boltzmann's constant. According to the general method of reductive perturbation theory, we introduce the stretched variables

$$
\tau=\varepsilon^{3 / 2} t, \quad \xi=\varepsilon^{1 / 2}(x-\lambda t),
$$

where $\lambda$ is the unknown phase velocity, to be determined later, and all the physical quantities appeared in (1) are expanded as power series in $\varepsilon$ about the equilibrium values as

$$
\begin{aligned}
& n=1+\varepsilon n_{1}+\varepsilon^{2} n_{2}+\ldots \\
& u=\varepsilon u_{1}+\varepsilon^{2} u_{2}+\ldots \\
& p=1+\varepsilon p_{1}+\varepsilon^{2} p_{2}+\ldots \\
& \phi=\varepsilon \phi_{1}+\varepsilon^{2} \phi_{2}+\ldots \\
& n_{e}=1+\varepsilon n_{e 1}+\varepsilon^{2} n_{e 2}+\ldots
\end{aligned}
$$

$\varepsilon$ represents the amplitude of the perturbation. We impose the boundary conditions that as $|\xi| \rightarrow \infty, n=n_{e}=p=1, u=$ $u_{0}=\phi=0$. Substituting (2) and (3) into the system of equations (1) and equating coefficients of like powers of $\varepsilon$. Then, from the lowest, second-order equations in $\varepsilon$. and by eliminating the second order perturbed quantities $n_{2}, u_{2}, p_{2}$, $n_{e 2}$ and $\phi_{2}$, we obtain the following $\mathrm{KdV}$ equation for the first-order perturbed potential:

$$
\begin{aligned}
& \frac{\partial}{\partial \tau} \phi_{1}+\left(\frac{6 \sigma+1}{\sqrt{3 \sigma+1}}\right) \phi_{1} \frac{\partial}{\partial \xi} \phi_{1}+ \\
& +\left(\frac{1}{2 \sqrt{3 \sigma+1}}\right) \frac{\partial^{3}}{\partial \xi^{3}} \phi_{1}=0 .
\end{aligned}
$$

\section{COMPUTERIZED SYMBOLIC COMPUTATION METHOD}

For a given partial differential equation in $\phi_{1}(\xi, \tau)$

$$
H\left(\phi_{1}, \frac{\partial \phi_{1}}{\partial \tau}, \frac{\partial \phi_{1}}{\partial \xi}, \frac{\partial^{2} \phi_{1}}{\partial \xi^{2}}, \ldots\right)=0 .
$$

In a travelling frame of reference

$$
\phi_{1}(\xi, \tau)=\Phi(\eta)=\Phi(\xi-v \tau)
$$

we may transform the partial differential equation into an ordinary differential equation

$$
H\left(\frac{\partial \Phi}{\partial \eta}, \frac{\partial^{2} \Phi}{\partial \eta^{2}}, \ldots\right)=0 .
$$

Step 1. Reduce partial differential equation (5) to the ordinary differential equation (6) by considering the wave transformation $\phi_{1}(\xi, \tau)=\Phi(\eta), \eta=\xi-v \tau$.

Step 2. Expand the solution of Eq. (6) in the form

$$
\phi_{1}(\xi, \tau)=\Phi(\eta)=\sum_{i=0}^{n} a_{i} \varphi^{i},
$$

where the new variable $\varphi(\eta)$ is a solution of the following ordinary differential equation

$$
\frac{d \varphi}{d \eta}=\varepsilon \sqrt{\sum_{j=0}^{r} c_{j} \varphi^{j}}, \quad \varepsilon= \pm 1 .
$$

Step 3. Determine the parameters $n$ and $r$. Substituting (8) into (6) and balancing the highest derivative term with the nonlinear terms, we then obtain a relation for $n$ and $r$, from which the different possible values of $n$ and $r$ can be obtained. These values lead to the different series expansions of the exact solutions. For $\mathrm{KdV}$ equation (4), we have $r=n+2$. 
If we take $n=2, r=4$, we have

$$
\begin{gathered}
\Phi(\eta)=a_{0}+a_{1} \varphi+a_{2} \varphi^{2} \\
\frac{d \varphi}{d \eta}=\varepsilon \sqrt{c_{0}+c_{1} \varphi+c_{2} \varphi^{2}+c_{3} \varphi^{3}+c_{4} \varphi^{4}}
\end{gathered}
$$

Step 4. Determine parameters $a_{i}(i=0,1, \ldots, n)$ and $c_{j}(j=0$, $1, \ldots, r)$. Substituting the expansions (9) into Eq. (6) and setting the coefficients of all powers of $\varphi^{i}$ and $\varphi^{i} \sqrt{\sum_{j=0}^{r} c_{j} \varphi^{j}}$ to zero, we will get a system of algebraic equations, from which the above parameters can be found explicitly.

Step 5. Solve Eq. (8). Substituting the parameters $c_{j}(j=0$, $1, \ldots, r$ ) obtained in Step 4 into Eq. (9), we can then obtain all the possible solutions. We remark here that the solutions of Eq. (5) depend on the explicit solvability of Eq. (9). The solution of the system of algebraic equations will be getting a series of fundamental solutions such as polynomial, exponential, soliton, rational, triangular periodic.

\section{EXPLICIT EXACT SOLUTIONS FOR THE KdV EQUATION}

Using the symbolic software package Maple, we obtain the following solutions:

$$
\begin{aligned}
& \Phi(\eta)=a_{0}-\frac{3\left((6 \sigma+1) a_{0}-v \sqrt{3 \sigma+1}\right) \operatorname{sech}^{2}\left(\frac{1}{\sqrt{2}} \eta \sqrt{-6 \sigma a_{0}-a_{0}+v \sqrt{3 \sigma+1}}\right)}{6 \sigma+1} \\
& \Phi(\eta)=a_{0}+\frac{3\left((6 \sigma+1) a_{0}-v \sqrt{3 \sigma+1}\right) \tan ^{2}\left(\frac{1}{2} \eta \sqrt{-6 \sigma a_{0}-a_{0}+v \sqrt{3 \sigma+1}}\right)}{12 \sigma+2}, \\
& \Phi(\eta)=a_{0}-\frac{3\left((6 \sigma+1) a_{0}-v \sqrt{3 \sigma+1}\right) \tanh ^{2}\left(\frac{1}{2} \eta \sqrt{6 \sigma a_{0}+a_{0}-v \sqrt{3 \sigma+1}}\right)}{12 \sigma+2}, \\
& \Phi(\eta)=a_{0}+\frac{3\left((6 \sigma+1) a_{0}-v \sqrt{3 \sigma+1}\right) \operatorname{csch}^{2}\left(\frac{1}{\sqrt{2}} \eta \sqrt{v \sqrt{3 \sigma+1}-(6 \sigma+1) a_{0}}\right)}{6 \sigma+1} \\
& \Phi(\eta)=\frac{v \sqrt{3 \sigma+1}}{6 \sigma+1}-\frac{6}{\eta^{2}(6 \sigma+1)}, \\
& \Phi(\eta)=a_{0}+\frac{3 m^{2}\left(v-a_{0} A\right) \mathrm{cn}\left(\frac{1}{2} \eta \sqrt{\frac{a_{0} A-v}{B\left(1-2 m^{2}\right)}}\right)^{2}}{A\left(2 m^{2}-1\right)}, \quad 0 \prec \frac{-a_{0} A+v}{4 B},
\end{aligned}
$$

where $m$ is a modulus.

$$
\Phi(\eta)=a_{0}+\frac{3\left(a_{0} A-v\right) \operatorname{dn}\left(\frac{1}{2} \eta \sqrt{\frac{a_{0} A-v}{B\left(m^{2}-2\right)}}\right)^{2}}{A\left(m^{2}-2\right)}, \quad 0 \prec \frac{-a_{0} A+v}{4 B},
$$




$$
\Phi(\eta)=a_{0}+\frac{3 m^{2}\left(v-a_{0} A\right) \operatorname{sn}\left(\frac{1}{2} \eta \sqrt{\frac{a_{0} A-v}{B\left(m^{2}+1\right)}}\right)^{2}}{A\left(m^{2}+1\right)}, \quad \frac{-a_{0} A+v}{4 B} \prec 0,
$$

where $a_{0}$ is an arbitrary constant, $A$ and $B$ are given by

$$
A=\frac{6 \sigma+1}{\sqrt{3 \sigma+1}}, \quad B=\frac{1}{2 \sqrt{3 \sigma+1}},
$$

and the Weierstrass elliptic doubly periodic type solution

$$
\Phi(\eta)=\frac{v}{A}-\frac{32^{2 / 3} B c_{3}^{2 / 3}}{A} \wp\left(\frac{\sqrt[3]{c_{3}}}{2^{2 / 3}} \eta ;-c_{1} \frac{2^{2 / 3}}{\sqrt[3]{c_{3}}} ;-c_{0}\right),
$$

where $c_{0}, c_{1}$ and $c_{3}$ are arbitrary constants.

\section{DISCUSSION AND CONCLUSION}

Numerical studies have been made for small amplitude ion-acoustic waves in unmagnetized collisionless plasma consisting of a mixture of warm ion-fluid and isothermal electrons. We have derived the Korteweg-deVries equation by using the reductive perturbation method, a symbolic computational traveling wave method used in obtaining a series of exact solutions of the $\mathrm{KdV}$ equation. The solution (10) is a solitary wave solution, in Fig. (1), a profile of the bell-shaped solitary pulse is obtained for solution (10). Figure 1 shows that the amplitude and width decreases with the increase of $\sigma$. Also solutions $(11,12)$ are singular type periodic solutions that develop a solution with singularity at a finite point called "blowup" of solutions as in Fig. 2a, in Fig. 2b, for some values of $a_{0}$ the pulse does not vanish at infinity although it has a localized form. Therefore, the arbitrary value $a_{0}$ plays a role in obtaining a pulse-shaped localized solution [18]. Solutions $(13,14)$

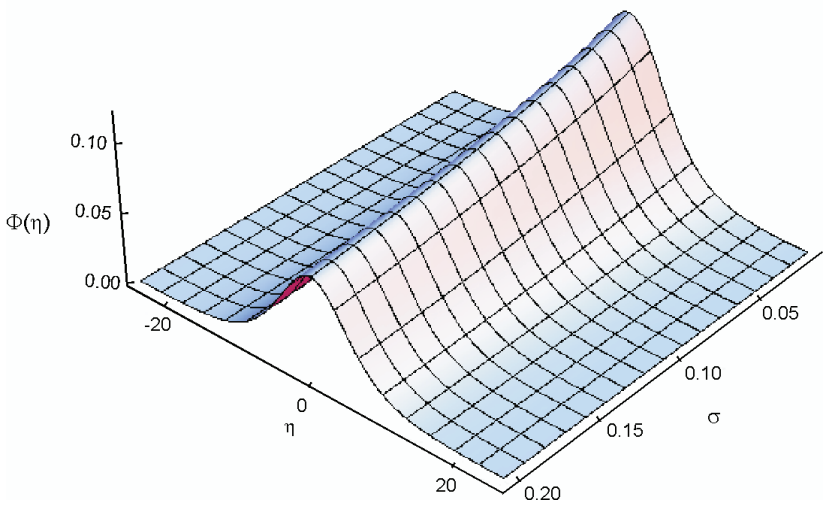

Fig. 1. The bell-shaped soliton solution for expression (10) for $a_{0}=0.008$ and $v=0.04$

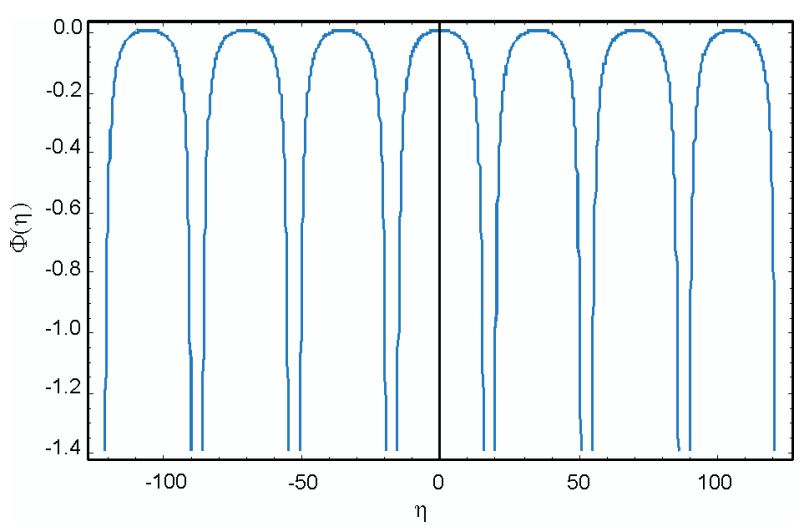

Fig. 2a. The blowup solution for expression (12) for $a_{0}=0.008$, $\sigma=0.03$ and $v=0.04$

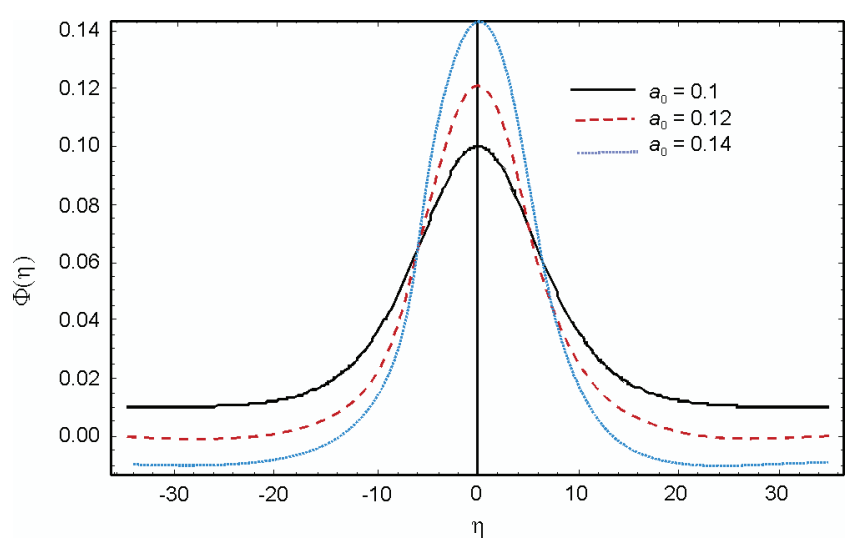

Fig. 2b. The profile of localized pulses for expression (12) for different values of $a_{0}$ for, $\sigma=0.001$ and $v=0.04$

lead to the propagation of explosive pulses as depicted in Fig. 3. The rational solution (14) may be helpful to explain certain physical phenomena. Because a rational solution is a disjoint union of manifolds, particle systems describing 


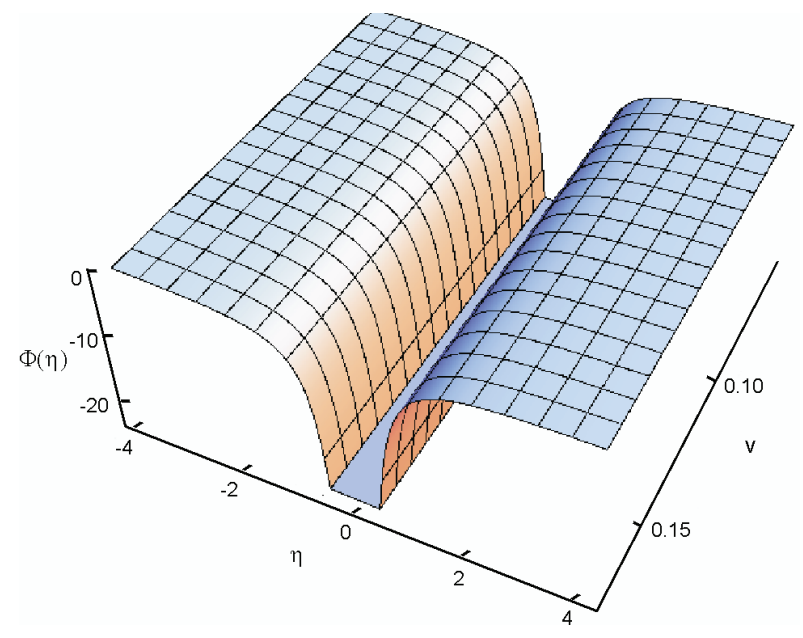

Fig. 3. The explosive solution for expression (14) for $a_{0}=0.008$ and $\sigma=0.03$

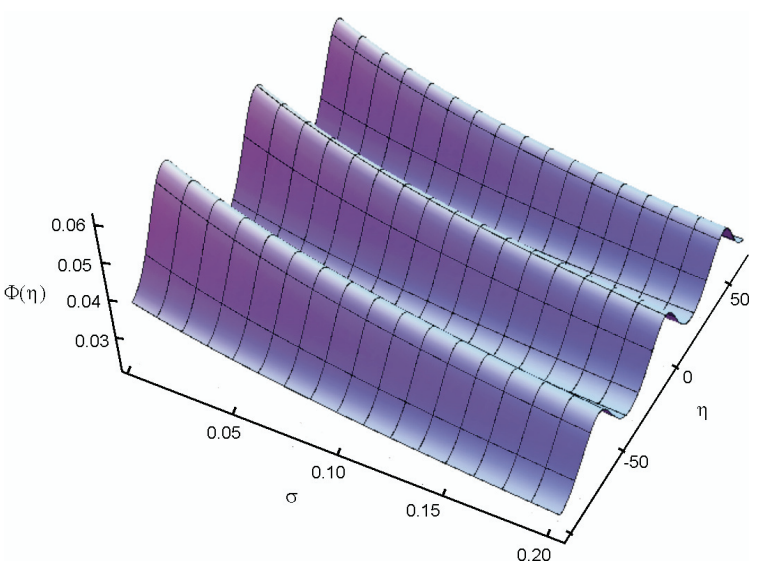

Fig. 4. The Jacobi elliptic doubly periodic solution for expression (17) for $m=2, a_{0}=0.008$ and $v=0.04$.

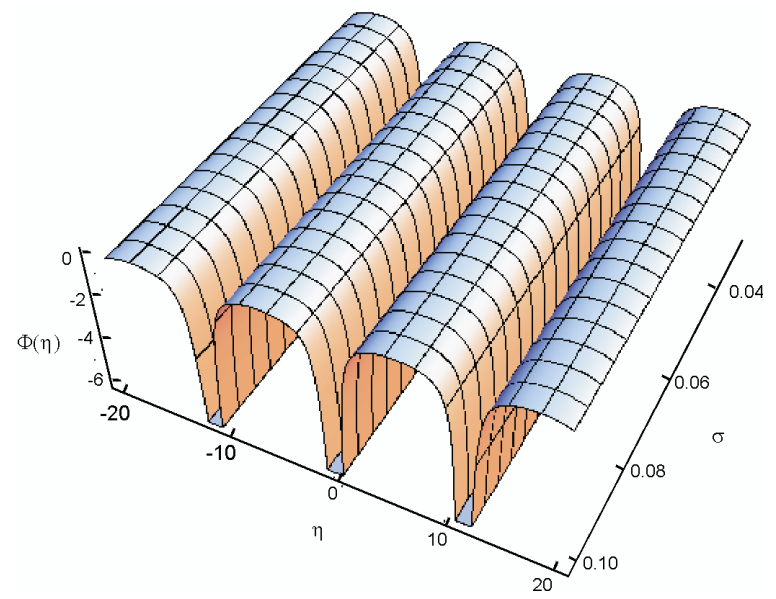

Fig. 5. Weierstrass elliptic doubly periodic type solution (20) for $c_{0}=-1, c_{1}=1, c_{3}=0.1$ and $v=0.04$ the motion of a pole of rational solutions for a $\mathrm{KdV}$ equation were analyzed [19]. Equations (15)-(17) are three Jacobi elliptic doubly periodic wave solutions as in Fig. (4), when $m \rightarrow 1$, solutions (15), (16) reduces to (10) while (17) reduces to (11). On the other hand, equation (18) gives the Weierstrass elliptic doubly periodic type solution as depicted in Fig. 5.

To sum up, it has been found that the amplitude and width of the ion-acoustic waves as well as parametric regime where the solutions can exist is sensitive to $\sigma$. Moreover, new exact solutions for (KdV) equation have been obtained and some of these solutions have been reported. It may be important to explain some physical phenomena in some plasma environments, such as ionosphere.

\section{References}

[1] G. Whitham, Linear and nonlinear waves. New York, Wiley (1974).

[2] R. Davidson, Methods in nonlinear plasma theory. New York, Academic Press (1972).

[3] S. K. El-Labany, J. Plasma Phys. 50, 495 (1993)..

[4] M. Sokała, J. Computational Methods in Science and Technology 14(2), 111 (2008).

[5] H. Washimi, T. Taniuti, J Phys Rev Lett. 17. 996 (1966).

[6] T. Taniuti, C. Wei, J Phys Soc Jpn 24, 491 (1968).

[7] S.A. Elwakil, E.K. El-Shewy, R. Sabry, Int. J. Nonlinear Sci. Numer. Simulation 5, 403 (2004).

[8] S.A. Elwakil, M.T. Attia, M.A. Zahran, E.K. El-Shewy, H.G. Abdelwahed, Z. Naturforsch. 61a, 316 (2006).

[9] M.J. Ablowitz, P.A. Clarkson, Solitons, nonlinear evolution equations and inverse scattering. Cambridge, Cambridge University Press (1991).

[10] W. Malfliet, Solitary wave solutions of nonlinear wave equations. American Journal of Physics 60(7), 650-654 (1992).

[11] E. Fan, Phys. Lett. A 277, 212 (2000).

[12] S.A. Elwakil, S.K. El-Labany, M.A. Zahran, R. Sabry, Phys. Lett. A 299, 179 (2002).

[13] M.A. Abdou, Applied Mathematics and Computation 190, 988-996 (2007).

[14] J.H. He, M.A. Abdou, New periodic solutions for nonlinear evolution equations using Exp-function method. Chaos, Solitons \& Fractals 2007;34:1421.

[15] M.A. Abdou, Chaos Soliton Fractals 31, 95-104 (2007).

[16] M.A. Abdou, A. Elhanbaly, Communications in Nonlinear Science and Numerical Simulation 12, 1229-1241 (2007).

[17] E. Fan, Chaos, Solitons and Fractals 16, 819 (2003).

[18] W.M. Moslem, R. Sabry, U.M. Abdelsalam, I. Kourakis, P.K. Shukla, New Journal of Physics 11, 033028 (2009).

[19] M. Adler, J. Moser, Commun. Math. Phys. 19, 1 (1978) 

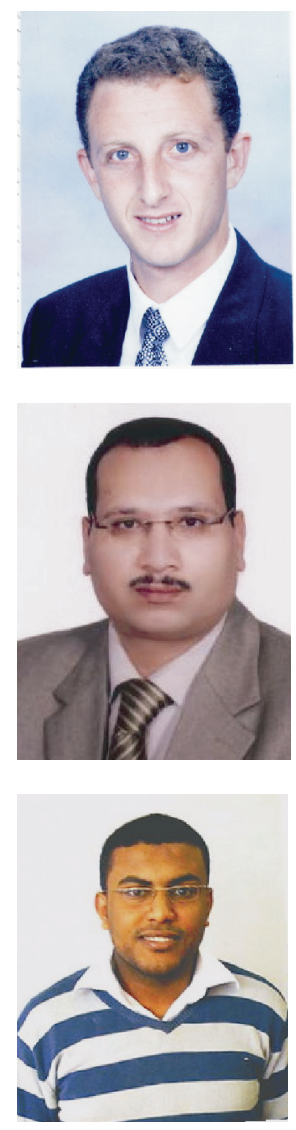

Emad El-Shewy. Assistant Professor of Theoretical Physics, Theoretical Physics Group, Department of Physics, Faculty of Science, Mansoura University, Mansoura, Egypt. Graduated in Physics in 1991. Since then he has been working in Mansoura University. He earned the PhD in nonlinear plasma physics in 2002. His research interests concern theoretical and computational physics. He is author of about 45 papers appearing in journals and international conference proceedings.

Hesham G. Abdelwahed. PhD in theoretical Physics, Theoretical Physics Group, Department of Physics, Faculty of Science, Mansoura University, Mansoura, Egypt. Graduated in Physics in 1998. Since then he has been working in Mansoura University. He earned the PhD in nonlinear plasma physics in 2007. His research interests concern theoretical and computational physics. He is author of about 10 papers appearing in journals and international conference proceedings.

HAMDi M. AbD-El-HaMid was born on December 31, 1984. MSc. Student of theoretical physics, graduated in Physics in 2006. Since then he has been working in the Department of Physics, Faculty of Science, Mansoura University, Mansoura, Egypt. His fields of interest are nonlinear phenomena, plasma physics, and algebraic methods of investigation of the exact solutions of nonlinear evolution equations. 